barig is. Haar prospectieve onderzoek toont aan dat routinematige screening naar ictale hartritmestoornissen bij het Dravetsyndroom niet effectief is; er werden geen klinisch relevante ritmestoornissen gevonden. Wel vond zij vaker een ictale QTc-verlenging bij de patiënten met het Dravetsyndroom dan bij de controles. Dit zou mogelijk het risico op gevaarlijke ritmestoornissen kunnen verhogen. Of dit werkelijk het geval is en of dit implicaties heeft voor het SUDEP-risico vergt langere termijn follow-up van dit cohort. Een andere belangrijke nevenbevinding was het grote aantal (waarschijnlijk tonisch-clonische) aanvallen, voornamelijk 's nachts, dat gemist werd door de verzorgers. Optimaliseren van aanvalsdetectie is daarom naast aanvalscontrole de beste interventie om het SUDEPrisico zo veel mogelijk te beperken in deze hoog-risico groep (van der Lende et al., 20I8).

\section{Referenties}

Harden C, Tomson T, Gloss D, Buchhalter J, Cross JH,

Donner E et al. (2017) Practice guideline summary:

Sudden unexpected death in epilepsy incidence rates and risk factors: Report of the Guideline Development, Dissemination, and Implementation Subcommittee of the American Academy of Neurology and the American Epilepsy Society. Neurology 2017; 88:1674-1680.

Liebenthal JA, Wu S, Rose S, Ebersole JS, Tao JX (20I5)

Association of prone position with sudden unexpected death in epilepsy. Neurology 2015; 84: 703-9.

Shmuely S, Sisodiya SM, Gunning WB, Sander JW, Thijs

RD (20r6a) Mortality in Dravet syndrome: A review.

Epilepsy Behav 2016a; 64: 69-74.

Shmuely S, Surges R, Sander JW, Thijs RD (2or6b) Prone

sleeping and SUDEP risk: the dynamics of body positions in nonfatal convulsive seizures. Epilepsy Behav 2016b; 62:176-9.

Shmuely S, Thijs RD (20I9) Epilepsy and Heart Diseases. In: Mula M. The Comorbidities of Epilepsy. London, UK: Academic Press; 2019; 159-76.

Van der Lende M, Hesdorffer DC, Sander JW, Thijs RD

(2018) Nocturnal supervision and SUDEP risk at different epilepsy care settings. Neurology 2018; 91: e1508-e18.

\title{
Epilepsie met myoclonisch- atone aanvallen of het syndroom van Doose
}

Kinderen met het syndroom van Doose vertonen zowel klinisch als elektrofysiologisch specifieke kenmerken. In deze bijdrage worden de typische EEG-kenmerken van het Doose-syndroom geillustreerd aan de hand van een casus.

Sinds 1989 is het syndroom van Doose opgenomen in de ILAE-classificatie van epilepsiesyndromen. Aanvankelijk droeg het de naam 'Epilepsie met myoclonisch-astatische aanvallen'; sinds de revisie van de terminologie in 2010 is het gewijzigd in 'Epilepsie met myoclonisch-atone aanvallen (EMAA)، (Berg et al., 20I0). Het beeld werd in 1970 beschreven door Hermann Doose (Doose et al., I970). Volgens de ILAE classificatie van 2017 is het een gegeneraliseerd epilepsiesyndroom met gegeneraliseerde motorische en niet-motorische aanvallen.

\section{Casus}

Het betreft een vierjarige jongen met een debuutleeftijd van de epilepsie van drie-en-een-half jaar. Er traden enkele nachtelijke bilateraal tonisch-clonische aanvallen op. Later kwamen ook absences en atone aanvallen voor. De psychomotorische ontwikkeling was normaal, totdat enkele maanden voor het epilepsiedebuut een algemene ontwikkelingsen taalachterstand werd geconstateerd. Er waren geen koortsstuipen in de voorgeschiedenis. Een oom van het jongetje heeft op kinderleeftijd epilepsie gehad (gedetailleerdere informatie ontbreekt). Andere familieleden met epilepsie zijn niet bekend. Het neurologisch onderzoek, de MRI van de hersenen en genetisch onderzoek (CGH-array) lieten geen afwijkingen zien. Het genetisch onderzoek zal worden uitgebreid met een genpanel. Gezien de typische debuutleeftijd en de verschillende aanvalstypen werd gedacht aan Epilepsie met myoclonisch-atone aanvallen (EMAA). 


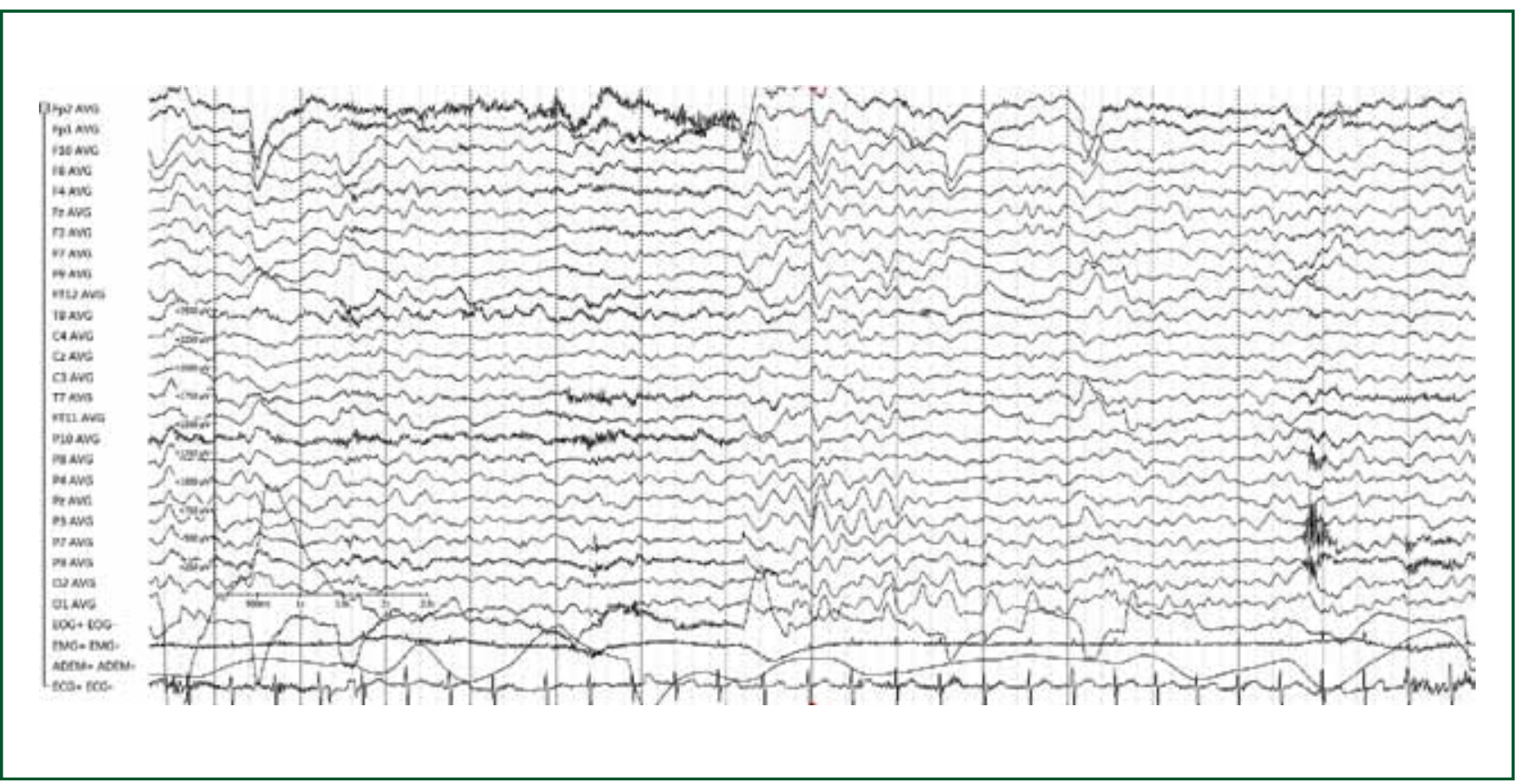

Figuur 1 Bi-pariëtale ritmische theta-activiteit. Weergegeven EEG in waak, met het gemiddelde (AVG) als referentie plus EOG, EMG, $\operatorname{ADEM}$ (haling) en ECG (Hp-filter 0,4 Hz; LP-filter $80 \mathrm{~Hz}$ ).

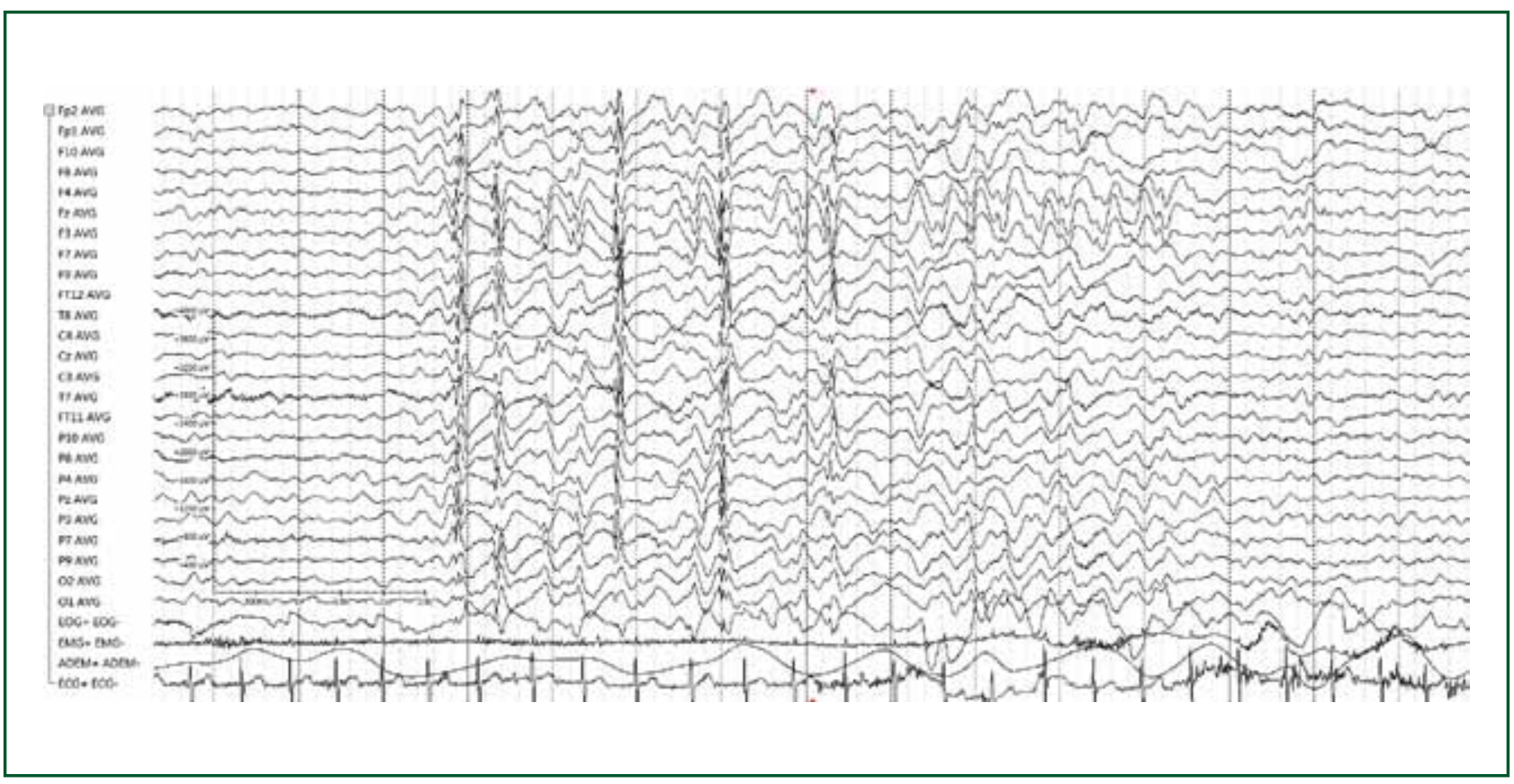

Figuur 2 Diffuse 2-2,5 Hz piekgolfcomplexen. Weergegeven EEG in waak, met het gemiddelde (AVG) als referentie, plus EOG, EMG, ADEM(haling) en ECG. (Hp-filter 0,4 Hz; LP-filter $80 \mathrm{H}$ ).

Het achtergrondpatroon van de 24-uurs video-EEG-registratie was normaal en er was geen sprake van lichtflitsgevoeligheid. Daarnaast trad bi-pariëtaal ritmische thetaactiviteit rond de $4 \mathrm{~Hz}$ op (figuur I) en diffuse, polymorfe piekgolf-, scherpe golf- en trage golfcomplexen met een frequentie van 2-2,5 Hz (figuur 2). Deze EEG-activiteit ging niet gepaard met klinische verschijnselen. Ictaal werden atypische absences geregistreerd met reeksjes diffuse piekgolfcomplexen met een frequentie rond de $2-2,5 \mathrm{~Hz}$, gepaard gaand met staken van de activiteit door de patiënt. In figuur 3 is het EEG weergegeven tijdens het optreden van de volgende klinische verschijnselen: vertraagde reactie, wegdraaien van de ogen en patiënt kan testwoord niet herhalen. De myoclonisch-atone aanvallen traden op met korte heffing van de armen gevolgd door hoofdknikjes of door de knieën zakken. In het EEG werd daarbij een diffuse piek waargenomen met achtereenvolgens een trage golf en afvlakking van de curve (figuur 4).

Achtergrond: anamnese en kliniek

Typisch voor het syndroom van Doose is een debuutleeftijd van zes maanden tot zes jaar, met een piek tussen twee 


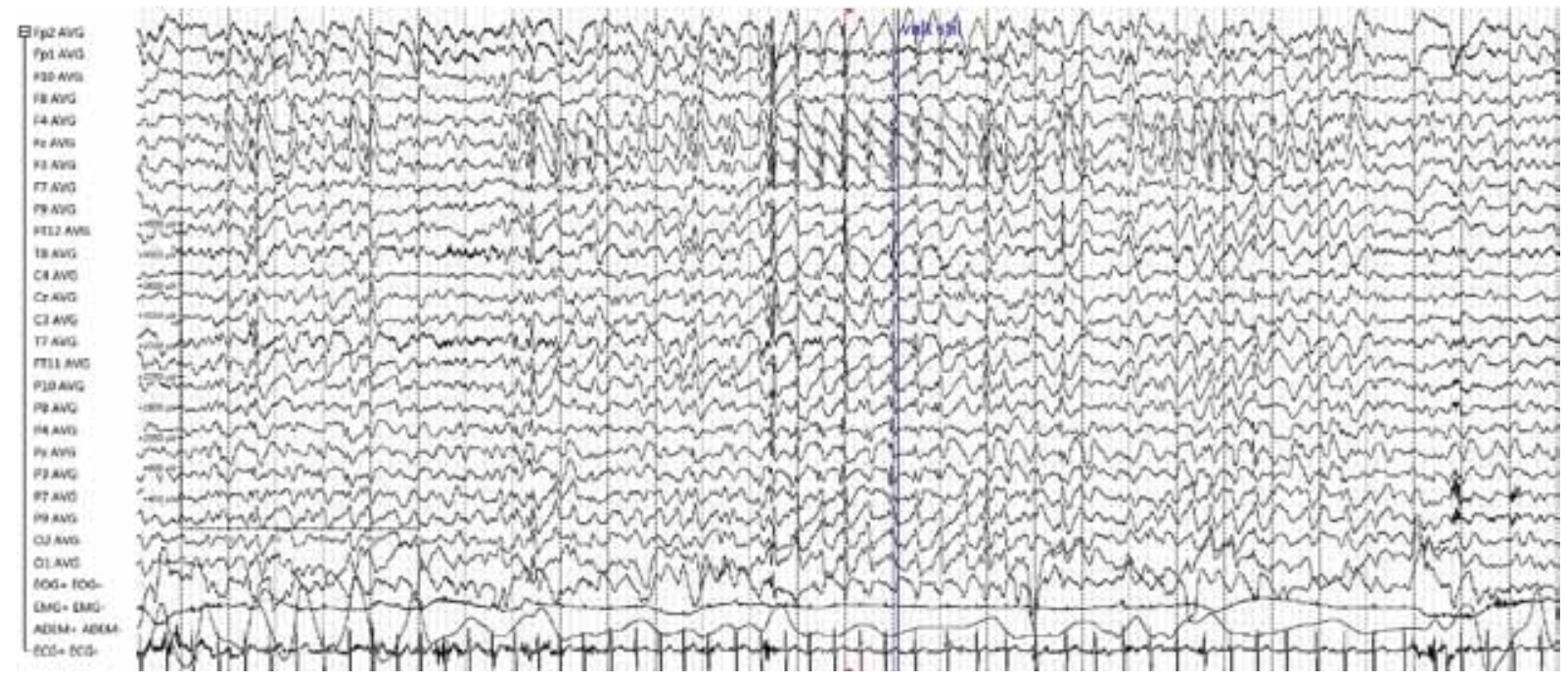

Figuur 3 Diffuse 2-2,5 Hz piekgolfcomplexen met een duur van ongeveer 18 seconden, geclassificeerd als atypische absence. Weergegeven EEG in waak met het gemiddelde (AVG) als referentie plus EOG, EMG, ADEM(haling) en ECG. (Hp-filter 0,4 Hz; LP-filter 8o Hz).

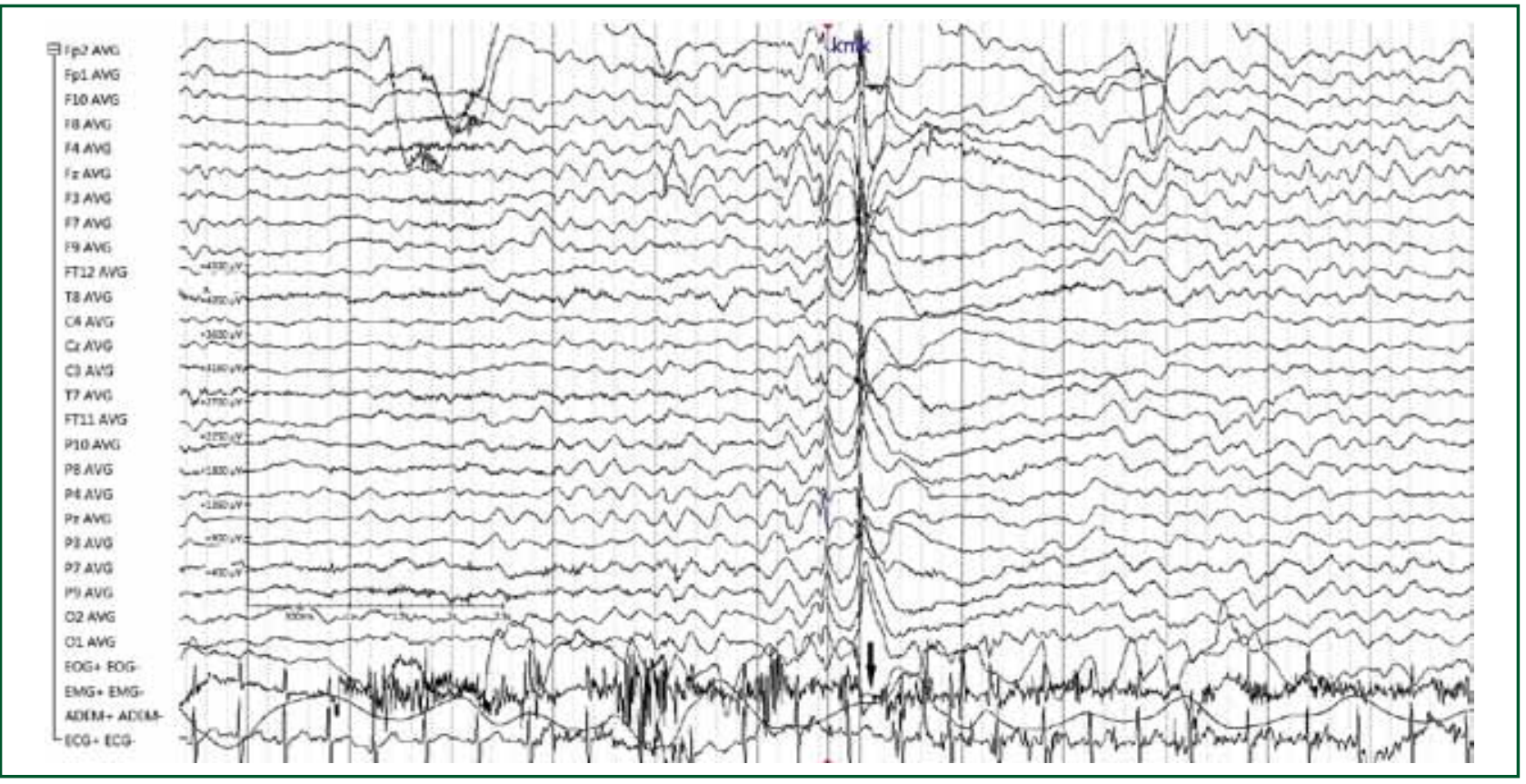

Figuur 4 Myclonisch-atone aanval met een diffuus piekgolfcomplex met achtereenvolgende afulakking in het EEG. Het begin van het hoofdknikje wordt door de pijl gemarkeerd en valt samen met een relatieve afulakking van het EMG waardoor de atone karakter van het knikje bevestigt wordt. Weergegeven het EEG in waak, met het gemiddlde (AVG) als referentie plus EOG, EMG, ADEM(haling) en ECG. (EMG kanaal: Hp-filter $2 \mathrm{~Hz}$, Gain gedubbeld; EEG kanalen: Hp-filter 0,4 Hz; LP-filter $80 \mathrm{~Hz}$ ).

en vier jaar. Neurologisch onderzoek en MRI van de hersenen tonen geen bijzonderheden. In de families van de kinderen komen koortsstuipen en epilepsie regelmatig voor. Er wordt een multifactoriële polygenetische overerving en etiologie voor de meerderheid van de patiënten aangenomen. Bij een gedeelte van de patiënten wordt een pathogene mutatie in een bekend epilepsiegen gevonden, waaronder het SLC6AI-gen (Routier et al., 2019).

Kenmerkend voor het syndroom zijn myoclonisch-atone aanvallen, bestaande uit myoclonieën in de schouders of armen met vervolgens een atone verslapping. De frequentie van de activiteit kan oplopen tot een status epilepticus. Daarnaast komen absences en bilateraal tonisch-clonische aanvallen voor.

Wat het optreden van tonische aanvallen bij EMAA betreft zijn de meningen verdeeld. Volgens Panayiotopoulos (2010) zijn tonische aanvallen een exclusiecriteria voor de diagnose maar volgens anderen kunnen tonische aanvallen bij 
patiënten met EMAA wel degelijk voorkomen, met name 's nachts (International League against Epilepsy 20I9; Doose et al.,I970; Neubauer et al., 2005). Focale aanvallen en epileptische spasmen pleiten tegen het syndroom.

In het algemeen is bij EMAA het achtergrondpatroon normaal. Bi-pariëtale theta-activiteit is vaak de eerste opvallende en kenmerkende verandering. Later treden interictaal diffuse (poly-) piekgolf- en scherpe golf- trage golfcomplexen op met een frequentie van 2-3 Hz. Focale afwijkingen pleiten tegen de diagnose.

Ictaal worden tijdens de myoclonisch-atone aanvallen diffuse pieken of polypieken gezien, gevolgd door een trage golf. De trage golf is het correlaat van de atone fase, wat door een afvlakking in het EMG bevestigd kan worden (International League against Epilepsy, 2019; Panayiotopoulos, 2010).

\section{Differentiaaldiagnose}

Indien alle bovengenoemde klinische en EEG-kenmerken aanwezig zijn is de diagnose EMAA niet moeilijk. Met name de combinatie van verschillende gegeneraliseerde aanvalstypes, waaronder myoclonisch-atone aanvallen, een blanco voorgeschiedenis en ontwikkeling en de typische gegeneraliseerde afwijkingen in het EEG (zoals hierboven beschreven), met ontbreken van focale afwijkingen, leidt tot de diagnose EMAA.

Differentiaal diagnostisch kan bij jonge kinderen met een normale ontwikkeling gedacht worden aan een andere gegeneraliseerde epilepsie zoals childhood absence epilepsy, gekenmerkt door absences en op deze jonge leeftijd in mindere mate tonisch-clonische aanvallen. De kenmerken myoclonisch-atone aanvallen en polypieken in het EEG komen hierbij niet voor.

De differentiatie met het Lennox-Gastaut-syndroom (LGS) kan klinisch soms lastig zijn, met name als er geen anamnese van eerdere epilepsiesyndromen is. Vaak is er bij LGS echter al een duidelijke ontwikkelingsachterstand en soms een genetische mutatie of afwijkingen op de MRI hersenen. $\mathrm{Bij}$ het LGS past in vergelijking met EMAA een breder spectrum van aanvalstypes. Een gestoord achtergrondpatroon en focale afwijkingen in het EEG pleiten tegen EMAA en wijzen diagnostisch op een LGS.

Nabeschouwing

Klinisch voldoet de hier beschreven casus bijna volledig aan de criteria voor EMAA. De patiënt is niet lichtflitsgevoelig en heeft geen koortsstuipen gehad, wat niet tegen de diagnose pleit. Het video-EEG (met EMG-kanaal) steunde de diagnose EMAA. Er worden de typische interictale afwijkingen in combinatie met twee bij het syndroom passende type aanvallen gezien, waaronder de kenmerkende myoclonisch-atone aanvallen. De anamnestisch beschreven bilateraal tonisch-clonische aanvallen passen in het aanvalsspectrum, deze werden echter niet geregistreerd. Na het starten van de medicatie verminderende de hoge aanvalsfrequentie en mogelijk ook de invloed hiervan op het cognitief functioneren.

\section{Conclusie}

Het onderscheiden van de verschillende kinderepilepsiesyndromen is van belang voor de juiste medicamenteuze behandeling, het doen van aanvullend onderzoek en de prognose. Het video-EEG (met EMG-kanaal) is hierbij van grote toegevoegde waarde.

\section{Referenties}

Berg AT, Berkovic SF, Brodie MJ, Buchhalter J, Cross JH et al. (2010) Revised terminology and concepts for organization of seizures and epilepsies: report of the ILAE Commission on Classification and Terminology, 20052009. Epilepsia 51 (4), 676-685.

Doose H, Gerken H, Leonhardt R, Völzke E, Völz C (I970) Centrencephalic myoclonic-astatic petit mal. Clinical and genetic investigation. Neuropadiatrie 2 (1), 59-78. International League against Epilepsy (2019) Epilepsy with myoclonic-atonic seizures. https:||www.epilepsydiagnosis.org/syndrome/epilepsy-myoclonic-atonic-overview.html. Neubauer BA, Hahn A, Doose H, Tuxhorn I (2005). Myoclonic-astatic epilepsy of early childhood--definition, course, nosography, and genetics. Advances in neurology 95, 147-155.

Panayiotopoulos CP (20I0) A Clinical Guide to Epileptic Syndromes and their Treatment, Revised Second Edition ed. Springer-Verlag London, London.

Routier L, Verny F, Barcia G, Chemaly N, Desguerre I, Colleaux L, Nabbout R (20rg) Exome sequencing findings in 27 patients with myoclonic-atonic epilepsy: Is there a major genetic factor? Clinical genetics 96 (3), 254-260.

\section{Hebt u de website van het Epilepsiefonds al bekeken? www.epilepsie.nl}

